\title{
ESSENTIAL EXTENSIONS AND INTERSECTION THEOREMS
}

\author{
W. SCHELTER
}

ABSTRACT. If $R$ is right and left noetherian, primitive factor rings are artinian, and $R$ is right fully bounded, then a simple proof is given to show that finitely generated essential extensions of right artinian modules are artinian. An immediate corollary is that $\bigcap_{n=1}^{\infty} J^{n}=0$ for such a ring.

Let $R$ be a ring with 1 and $J$ its Jacobson radical. Recall that a ring is right fully bounded if essential right ideals of prime factor rings contain nonzero two-sided ideals. That rings satisfying a polynomial identity are right fully bounded is shown in [1]; indeed essential right ideals contain central elements.

Jategaonkar [3] has recently proved that if $R$ is a right and left noetherian, right and left fully bounded ring, then finitely generated essential extensions of artinian modules are artinian. His proof relies heavily on some very complicated and difficult results also in [3]. Our purpose is to present a short elementary proof of the following theorem, which has Jategaonkar's result as an immediate consequence. We note, however, all known examples of right and left noetherian rings which are right fully bounded, are also left fully bounded and so our Theorem may not be more general. Implications of the Theorem for the intersections of the powers of ideals other than the radical are given in [4].

Theorem. If $R$ is right and left noetherian, left ${ }^{1}$ primitive factor rings are artinian, and $R$ is right fully bounded then f.g, essential extensions of right artinian modules are right artinian.

Before proving the Theorem we note that the sum of the injective hulls of simple right $R$-modules is faithful (indeed an injective cogenerator for $\operatorname{Mod} R$ ), yet every f.g. submodule of this sum will be artinian, and so annihilated by $J^{n}$ for some $n$. As Jategaonkar notes, this yields the following

Corollary. If $R$ is as above, $\bigcap_{n=1}^{\infty} J^{n}=0$.

Received by the editors May 17, 1974 and, in revised form, August 6, 1974 and October 7, 1974 .

AMS (MOS) subject classifications (1970). Primary 16A46, 13E05; Secondary 16A26, 16A38.

1 I would like to thank $T$. Lenagan for pointing out to me that we may drop the assumption on left primitive factor rings, since the sequence of ideals $Q_{i}$ in our proof may be chosen to be right primitive by using the ideas of [4].

Copyright $\odot$ 1975. American Mathematical Society 
We require the following generalization of Nakayama's lemma.

Lemma. If $R$ is any ring, $R^{M}$ a finitely generated $R$ module, then there exists a left primitive ideal $Q$ such that $Q M \subsetneq M$.

Proof. Take $\sum_{i=1}^{n} R m_{i}=M$ such that $m_{1} \notin \sum_{i=2} R m_{i}=X$. Then $M \rightarrow$ $M / R m_{1} \cap X \simeq R \bar{m}_{1} \oplus \bar{X} \rightarrow S$ where $S$ is a simple homomorphic image of $R \bar{m}_{1}$. If $g: M \rightarrow S$ as above, then $g\left(\operatorname{ann}_{R} S \cdot M\right)=\operatorname{ann}_{R} S g(M)=0$, so $\left(\operatorname{ann}_{R} S\right) \cdot M \neq$ $M$,

Proof of the Theorem. Let $M_{R}$ be f.g., and an essential extension of an artinian module $A_{R}$. Let $X_{R}$ be a maximal artinian extension of $A_{R}$ in $M_{R}$, and suppose $X \neq M$. Take $M_{R}^{\prime} \supsetneqq X_{R}$ in $M$ such that ann ${ }_{R} M^{\prime} / X$ is maximal among such annihilator ideals, and write ann ${ }_{R} M^{\prime} / X=P$ since it is clearly prime. $P / a^{a n n}{ }_{R} M^{\prime}$ is a f.g. right $R / \operatorname{ann} X_{R}$ module, and the latter is artinian since it cont ains a finite product of primitives, namely the annihilators of the simple factor modules in a composition series for $X_{R}$. Take a sequence of left primitive ideals $Q_{1}, Q_{2}, \ldots$ by the Lemma such that

$$
Q_{i} Q_{i-1} \cdots Q_{1}\left(P / \operatorname{ann}_{R} M^{g}\right) \subsetneq Q_{i-1} \cdots Q_{1}\left(P / \operatorname{ann}_{R} M^{i}\right) .
$$

Thus $Q_{k} Q_{k-1} \cdots Q_{1} P \subseteq$ ann $_{R} M$. If $Q_{i} \subseteq P$ for some $i$ we are done, since $R / P$ is then artinian, and if not then $0 \neq M Q_{k} \cdots Q_{1}+X / X$, and it has annihilator $P$, and so it is a faithful $R / P$ module (and hence not singular since the intersection of the annihilators of a finite set of generators would be essential and so contain a nonzero two-sided ideal). But $M^{\prime} Q_{k} \cdots Q_{1}$ has essential submodule $X \cap M^{\prime} Q_{k} \cdots Q_{1}$, so $M^{\prime} Q_{k} \cdots Q_{1} / X \cap M^{\prime} Q_{k} \cdots Q_{1}$ is a singular $R / P$ module.

We remark in closing that it is well known that left fully bounded together with left noetherian imply that left primitive factor rings are artinian. (See [2, Theorem] for a much more general and stronger statement or, in our case, simply argue as follows: if ${ }_{R} S$ is a simple faithful $R$-module, and $0 \neq s \in S$, then ann $R_{R}$ is not essential, so ann $R_{R} \cap X=0$ for some left ideal $X$, and thus $X$ contains an isomorphic copy of $S$ as a minimal left ideal. But prime left noetherian rings with a left minimal ideal are easily seen to be artinian.)

\section{BIBLIOGRAPHY}

1. S. A. Amitsur, Prime rings having polynomial identities with arbitrary coefficients, Proc. London Math. Soc. (3) 17 (1967), 470-486. MR 36 \#209.

2. R. Gordon and J. Robson, Krull dimension, Mem. Amer. Math. Soc. No. 133 (1973), p. 58.

3. A. Jategaonkar, Jacobson's conjecture and modules over fully bounded noe. therian rings, J. Algebra 30 (1974), 103-121. 
4. T. Lenagan, Artinian ideals in noetherian rings (to appear).

5. W. Schelter, Intersection theorems for some non-commutative noetherian rings (to appear).

DEP ARTMENT OF MATHEMATICS, UNIVERSITY OF ALBERTA, EDMONTON, ALBERTA, C.ANADA TGG 2G1

Current addre's: Department of Mathematics, Dalhousie University, Halifax, Nova Scotia, Canada 